\title{
Benign Metastasizing Leiomyoma: Rare Manifestation of a Frequent Pathology
}

\author{
Maria Inês Raposo1,3, Catarina Meireles², Mariana Cardoso³, Mariana Ormonde ${ }^{3}$, \\ Cristina Ramalho ${ }^{1}$, Mónica Pires ${ }^{1}$, Mariana Afonso², Almerinda Petiz ${ }^{1}$ \\ ${ }^{1}$ Department of Gynecology, Francisco Gentil Portuguese Oncology Institute, Porto \\ ${ }^{2}$ Department of Pathology, Francisco Gentil Portuguese Oncology Institute, Porto \\ ${ }^{3}$ Department of Gynecology, Hospital of Divino Espírito Santo of Ponta Delgada, São Miguel, Azores
}

\section{Problem Statement:}

Benign metastasizing leiomyoma $(\mathrm{BML})$ is a rare variant of uterine leiomyoma, characterized by multiple leiomyomatous lesions in distant locations, most commonly the lungs. Patients are usually asymptomatic and the disease is discovered incidentally. Histopathological confirmation is required for definitive diagnosis. Treatment of BML is controversial. The course of this disease is usually indolent, but requires close surveillance.

\section{Methods:}

Overview of two clinical cases of BML diagnosed in Portuguese Oncology Institute of Porto in the first semester of 2017 and review of the literature.

\section{Case Reports:}

\begin{tabular}{|c|c|c|}
\hline & Case Report 1 & Case Report 2 \\
\hline Identification & 49-year-old premenopausal woman & 48-year-old premenopausal woman \\
\hline Medical History & Total hysterectomy 10 years previously for uterine leiomyoma & Total hysterectomy 13 years earlier for uterine leiomyoma \\
\hline Clinical presentation & Asymptomatic. Incidental diagnosis. & Persistent cough. \\
\hline $\begin{array}{c}\text { Imaging findings: } \\
\text { Chest radiography } \\
\text { and computed } \\
\text { tomography (CT) }\end{array}$ & Miliary pattern
\end{tabular}

- PET

Positron emission tomography: Weak fluorodeoxyglucose (FDG) uptake in lung nodules.

\section{Diagnosis:}

CT-guided biopsy of a pulmonary nodule and review of uterine specimen previously resected.

Both specimens showed identical histopathology, immunohistochemical and cytogenetic characteristics.
Histopathologic examination

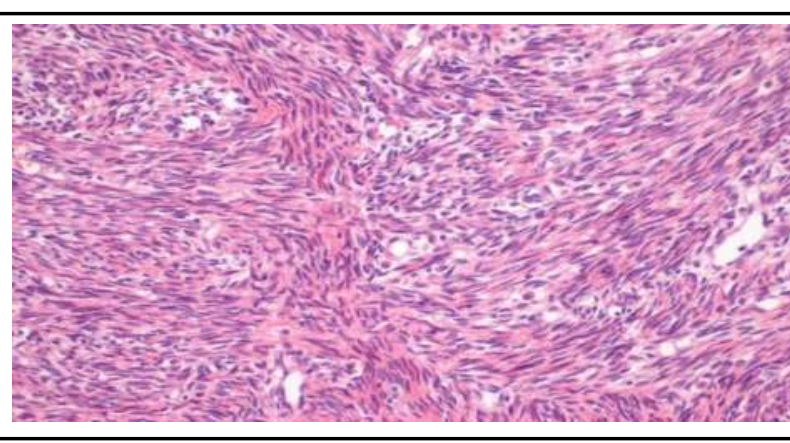

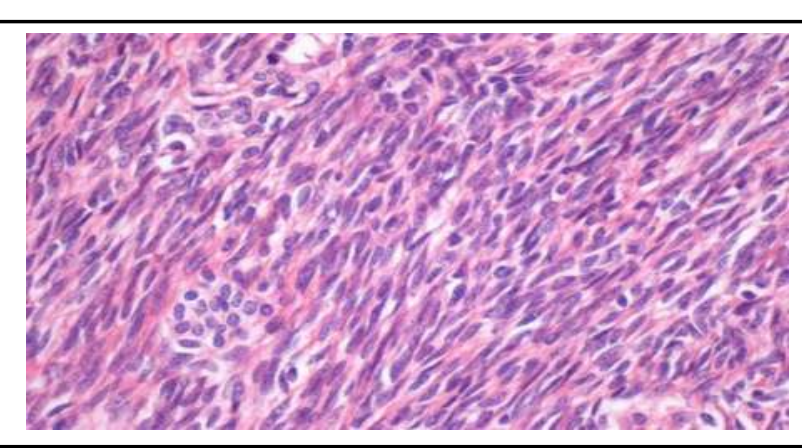

Spindle cells consistent with smooth muscle differentiation, without cellular atypia, necrosis nor mitotic figures.
Immunohistochemical staining

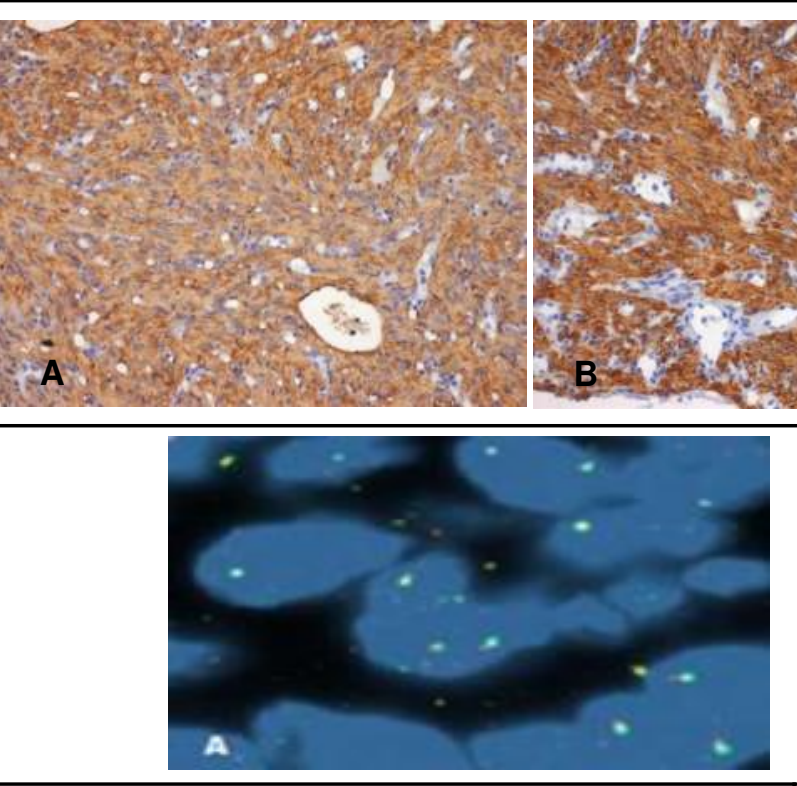

Cytogenetic study, using "fluorescence in situ hybridization" (FISH)

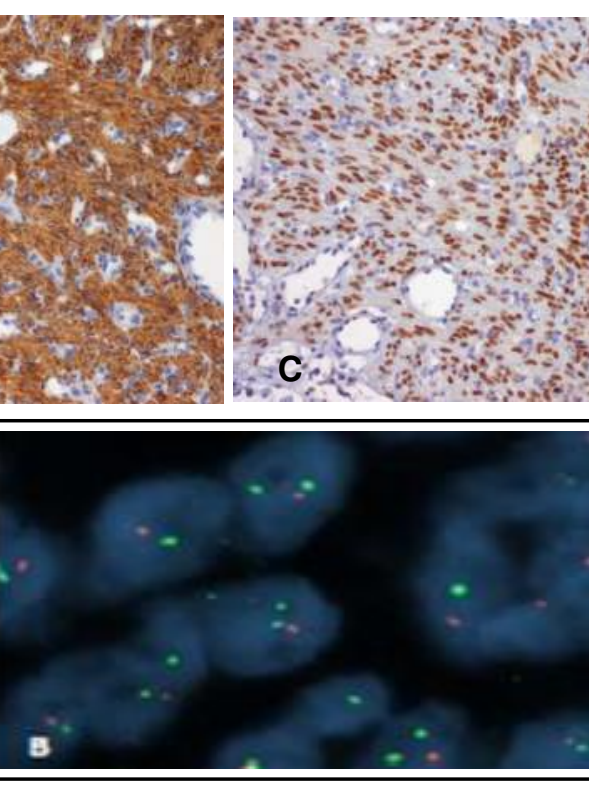

Bilateral salpingo-oophorectomy.
Treatment

Bilateral salpingo-oophorectomy followed by Letrozole.

Follow-up

6 months: No further development of the disease occured.
A. Smooth Muscle Actin;

B. Desmin;

C. Estrogen and Progesterone Receptors

\section{Conclusion:}

$\checkmark$ BML diagnosis is challenging and should be based on similar histopathological and immunohistochemical pattern between lung nodules and uterine leiomyoma. When the uterine specimen is not available for retrospective review, the presence of consistent chromosomal abnormalities (deletions of $19 q$ and $\mathbf{2 2 q}$ ) is highly suggestive of BML, allowing its individualization as a genetically distinct entity.

References:

[1] A. Nurettin et al "Benign Pulmonary Metastasizing Leiomyoma of the Uterus," J. Clin. Diagnostic Res., vol. 10, no. 9, pp. 3-5, 2016.

[2] S. Chen et al "Pulmonary benign metastasizing leiomyoma from uterine leiomyoma," World J. Surg. Oncol., vol. 11, no. 163, 2013.

[3] H. Ma et al "Benign pulmonary metastasizing leiomyoma of the uterus: A case report," Oncol. Lett., vol. 9, pp. 1347-1350, 2015.

[4] E. Barnas et al "Benign metastasizing leiomyoma : A review of current literature in respect to the time and type of previous gynecological surgery," PLoS One, pp. 1-12, 2017.

[5] K. Patton et al "Benign metastasizing leiomyoma: clonality, telomere length and clinicopathologic analysis.," Mod. Pathol., vol. 19, pp. 130-140, 2006.

[6] M. R. Nucci et al "Distinctive Cytogenetic Profile in Benign Metastasizing Leiomyoma: Pathogenetic Implications," Am. J. Surg. Pathol., vol. 31, no. 5, pp. 737-743, 